\title{
Zu diesem Heft - Max Weber: Zum 100. Todestag eines Unvergessenen
}

Anlässlich seines 100. Todestags wurde Max Weber in diesem Jahr reichlich geehrt. In Zeitungen und Rundfunk erschienen unzählige Beiträge mit dem Ziel, Leben und Werk des großen Denkers einem breiteren Publikum zugänglich zu machen. Die Fachöffentlichkeit nahm das Jubiläum zum Anlass, um sein Euvre in Vortragsreihen, Diskussionsrunden und Tagungen zu würdigen und der Frage nachzugehen, was wir aus seinen Analysen für die Herausforderungen und Probleme der Gegenwart lernen können. ${ }^{1}$

Angesichts dieser breiten Aufmerksamkeit und der Bedeutung, die Webers Konzepten und Begrifflichkeiten auch heute noch für die aktuelle Forschung und das Verständnis spätmoderner Gesellschaften zugesprochen wird, überrascht es, dass das Jubiläum in der sozialwissenschaftlichen Zeitschriftenlandschaft kaum Resonanz erfuhr. Mit Ausnahme des Online-Portals Soziopolis, das Max Weber einen Schwerpunkt widmete, scheint es von keiner der größeren Zeitschriften der Politikwissenschaften, Ökonomie, Philosophie oder Rechtswissenschaften, ja nicht einmal der Soziologie, als deren Mitbegründer er bekanntlich gilt, wahrgenommen worden zu sein.

In dieser Leviathan-Ausgabe möchten wir die lebhafte sozialwissenschaftliche Debatte, zu der Webers 100. Todesjahr Anlass gab, bei der es sich bislang aber überwiegend um Rede- und Diskussionsbeiträge handelte, um eine schriftliche Form der Erinnerung ergänzen. Dafür haben wir Texte ausgewählt, die mit Politik und Herrschaft, Kapitalismus, Bürokratie und Wissenschaft einige zentrale Themen seines Werks aufgreifen.

Neben Freiburg, Heidelberg und München, wo Max Weber jeweils Professuren innehatte, ist auch Berlin eine wichtige Station in Webers privatem wie akademischem Leben gewesen. Im Alter von fünf Jahren verlässt er mit seinen Eltern seine Geburtsstadt Erfurt, um in die Haupt- und Residenzstadt des deutschen Kaiserreichs zu ziehen, wo sein Vater zum besoldeten Stadtrat gewählt worden war. 1882 macht er sein Abitur am Kaiserin-Augusta-Gymnasium in Berlin-Charlottenburg. Für sein Studium verlässt er Berlin zeitweise, kehrt aber 1886 nach seinem juristischen Staatsexamen aus finanziellen Gründen ins Elternhaus zurück, wo er bis zu seiner Heirat eine »verlängerte >Postadoleszenz< [...] unter dem Dach des patriarchalischen Vaters und der seelisch dominanten Mutter verbringen

$1 \mathrm{Zu}$ nennen wären hier etwa Veranstaltungen an den Universitäten Heidelberg und München, an denen Weber studierte und/oder lehrte, am Max-Weber-Kolleg der Universität Erfurt und am Schader-Forum in Darmstadt (in Kooperation mit dem Lehrstuhl für Wirtschaftssoziologie der Universität Trier). Auf dem diesjährigen Kongress der Deutschen Gesellschaft für Soziologie wurde ein ganzer Nachmittag über Webers Vermächtnis diskutiert. 
muss $«^{2}$ - eine Phase, in der er außerordentlich produktiv ist. Er absolviert ein Referendariat, das er 1890 mit dem Zweiten Juristischen Staatsexamen abschließt. Währenddessen promoviert er an der Friedrich-Wilhelms-Universität zu Berlin, wo er anschließend auch habilitiert und 1893 zum außerordentlichen Professor für Handelsrecht und deutsches Recht ernannt wird. Mit seinem Ruf nach Freiburg ein Jahr später endet seine Berliner Zeit. An diese soll nun eine anlässlich des 100. Todestags errichtete Gedenktafel an der nach seiner Mutter benannten "Villa Helene " in Charlottenburg erinnern, wo - so heißt es in Gangolf Hübingers Laudatio - »Max junior 21 prägende Jahre seines Lebens [verbrachte] ${ }^{3}$ Die Inschrift der Tafel lautet:

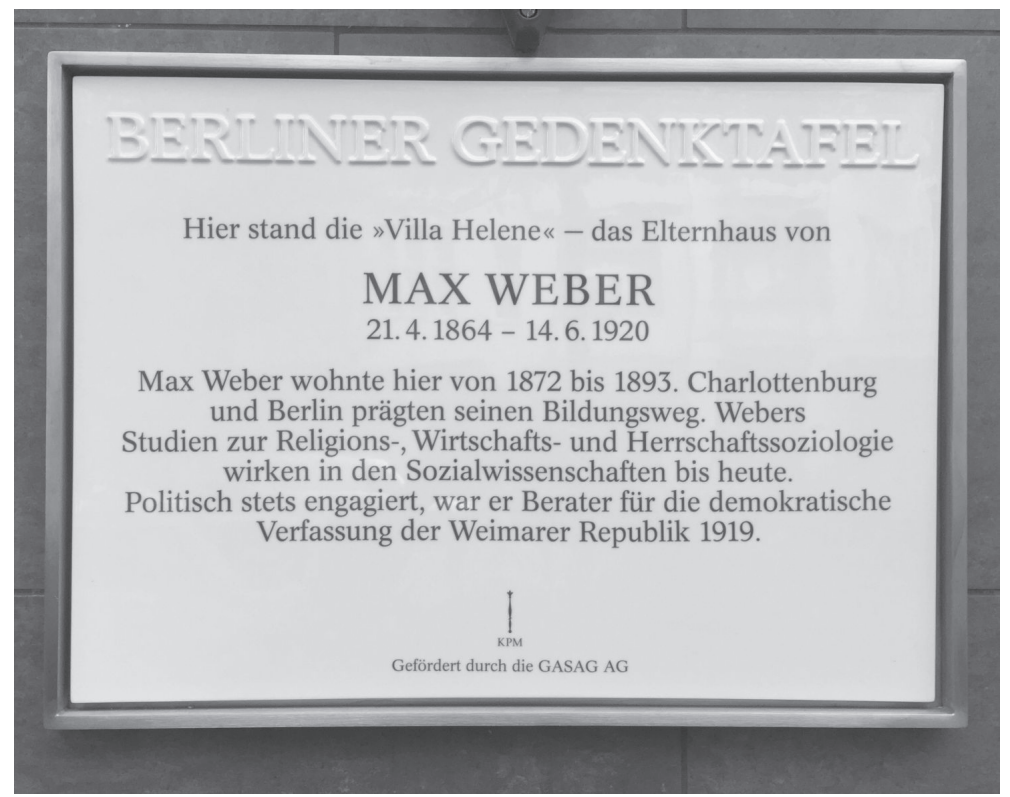

Quelle: eigenes Foto.

Weber war bekanntermaßen nicht nur Wissenschaftler, sondern zeit seines Lebens auch ein politisch interessierter und aktiver Mensch, der unter anderem - darauf verweist die Tafel im letzten Satz - als Mitglied des Verfassungsausschusses unter Hugo Preuß einen wichtigen Einfluss auf die Weimarer Verfassung ausübte. Doch worin bestand dieses politische Engagement und auf welchem Verständis von Demokratie beruhte es? Philip Manow stellt in seinem Beitrag fest, dass Weber in seiner Konzeption legitimer politischer Herrschaft etwas - zumindest aus heutiger

2 Hans-Peter Müller 2020. Max Weber. Werk und Wirkung. 2., aktualisierte und erweiterte Auflage. Köln: Böhlau, S. 26.

3 Siehe www.hiko-berlin.de/projekte/berliner-gedenktafeln/max-weber (Zugriff vom 22.10.2020). Die »Villa Helene« steht in der heutigen Leibnizstraße 21. 
Sicht - ganz Naheliegendes außer Acht lässt: die Tatsache, dass Herrschaft als legitim gilt, wenn ihr in Wahlen zugestimmt wird - die Demokratie also. Sein Entwurf einer plebiszitären Führerdemokratie beruhe gerade nicht auf den Prinzipien der Repräsentation, Delegation und Mandatierung, sondern auf der absoluten Gehorsamsbereitschaft gegenüber einem charismatischen Führer. Webers Vorstellung, dass Willensbildung von oben nach unten und nicht umgekehrt verläuft, führt Manow darauf zurück, dass er sich für die Analyse der Gegenwart vor allem auf die antike Demokratie beruft. Damit gerieten Parteien als zentrale Mobilisierungsakteure, aber auch die Bedeutung der Wahl und des Wahlrechts für deren innere Verfasstheit und parlamentarisches Wirken aus dem Blick - ein analytisches Versäumnis, das sein politisches Engagement auf verhängnisvolle Weise prägen sollte: Als Berater der Weimarer Verfassung trat Weber nachdrücklich für den direkt gewählten Reichspräsidenten und seine gegenüber dem Parlament umfassenden Auflösungs- und Notstandsvollmachten ein.

Webers Votum für die plebiszitäre Führerdemokratie ist nicht der einzige Grund, warum seine politische Haltung aus heutiger Sicht als bedenklich eingeschätzt werden könnte. Er war ein glühender Patriot und überzeugter Nationalist, sah sich subjektiv gar dazu geneigt, dem biologischen Erbgut einen nicht unerheblichen Einfluss auf die Lebensführung zuzuschreiben. Ist das nicht ausreichend, um ihn heute als nationalistisch und rassistisch abzulehnen?, fragt Hans-Peter Müller in seinem Beitrag und warnt zugleich vor voreiligen Schlüssen. Denn bei sorgfältiger Betrachtung werde offenbar, dass Weber sich im Zuge seiner soziologischen Auseinandersetzung mit »Nation " und »Rasse " zunehmend von diesen Begriffen distanziert und sie schließlich als wissenschaftlich unbrauchbar abgelehnt habe.

Den Begriff der "Nation" verweist Weber auf die Wertsphäre, weil sie - im Unterschied zur politischen Gemeinschaft oder zum Staat - keine institutionelle Ordnung auszuprägen vermag. Damit ergeht es ihr wie der Liebe und der Erotik. Deren Wert, das zeigt Ingrid Gilcher-Holtey in ihrem Beitrag, hat auch Weber erfahren, nachdem er sich lange Zeit kategorisch dagegen gewehrt hatte. Max und Marianne führten eine "Gefährtenehe", die sie ganz in den Dienst rationaler Werte, insbesondere ihres geistigen Schaffens stellten. Das »intellektuelle Paar « habe dem Erotisch-Sinnlichen bewusst entsagt und sich der Arbeit und dem politischen Engagement, besonders auch für die Rechte der Frauen, verpflichtet. Dieses rationale Lebensmodell sei jedoch herausgefordert worden, als die erotische Bewegung 1907/08 in das Heidelberger Milieu vordrang mit dem Ziel, die Geschlechterordnung durch die Freisetzung der Sexualität und Erotik der Frau zu transformieren. Diese Konfrontation habe letztlich dazu geführt, dass Weber seine ursprüngliche Position der Unterordnung der Erotik unter die Ethik der rationalen Selbststeuerung revidierte - nicht zuletzt deshalb, weil er den Wert des Sinnlichen selbst erfahren sollte.

Mit seiner Frau Marianne hatte sich Max Weber 1904 auf eine dreimonatige Amerikareise begeben. Er erhielt eine Einladung zu einem Kongress im Rahmen der Weltausstellung in St. Louis, Missouri, der er gerne folgte und die seinen Arbeits- und Schaffensdrang nach seiner mehrjährigen Neurasthenie-Erkrankung 
aufs Neue entfachte. Er sah darin eine willkommene Gelegenheit, sich selbst einen Eindruck von der Neuen Welt zu verschaffen. Fasziniert berichtet er den Daheimgebliebenen in Briefen von der fortgeschrittenen Gestalt, die der moderne Kapitalismus dort angenommen hatte, aber auch von den verheerenden Folgen für die Arbeiterschaft und die Umwelt, von Petroleum-Gestank, Qualm, Wasserverschmutzung und den Erkrankungen, die diese verursachten. Nur ein Jahr später schreibt er am Ende seiner Protestantischen Ethik, dass der Kapitalismus die Gesellschaftsordnung und Lebensweisen so lange prägen wird, »bis der letzte Zentner fossilen Brennstoffs verglüht ist «. Diesen Satz nimmt unsere Autorin Edith Hanke zum Anlass, um das Verhältnis von Kapitalismus und Umwelt bei Weber einer genaueren Betrachtung zu unterziehen. Wie hat Weber über die natürlichen Grundlagen der kapitalistischen Wirtschaftsweise nachgedacht? Ist eine ökologisch nachhaltige Lebensweise im modernen Kapitalismus überhaupt möglich? Dabei geht sie auch der Frage nach der Endlichkeit des Kapitalismus und nach Alternativ- beziehungsweise Gegenmodellen nach, die Weber und seine Zeitgenossen beschäftigten.

Neben einer begrenzten Rohstoffbasis und der Knappheit der Kohlevorräte sind es für Weber auch Prozesse der Rationalisierung und Bürokratisierung, die den liberalen, individualistisch geprägten Kapitalismus bedrohen. Die Bürokratie mit ihrem zentralen Prinzip berechenbarer Regelhaftigkeit betrachtet er zwar als eine wichtige Entstehungsvoraussetzung der kapitalistischen Wirtschaftsordnung, die "gerade diese >Berechenbarkeit des Erfolges ${ }^{4}$ und eine Orientierung an formalen Rationalitätskriterien verlange. Gleichzeitig beobachtet er jedoch eine Übermacht der Tendenz zur Bürokratisierung, die unternehmerische Freiheit, Verantwortung und Erfindergeist zu verdrängen und das gesellschaftliche Leben in einem »Gehäuse der Hörigkeit « zu erstarren drohe. Weber war nicht der Erste, der die umfassende Bürokratisierungstendenz der modernen Gesellschaft als etwas Bedrohliches wahrgenommen hat. Wie Pascale Cancik in ihrem Beitrag zeigt, reichen die Wurzeln dieser Kritik bis zum Beginn des 19. Jahrhunderts zurück. Der Begriff "Bürokratie« wurde in dieser Zeit als Schimpf- und Kampfwort geprägt und deutete bereits damals auf etwas Ungewolltes, Pathologisches hin, eine Wahrnehmung, die in der Gegenwart noch immer weit verbreitet ist. In ihrem Beitrag untersucht die Autorin die historische Entwicklung der negativen Semantiken des Bürokratiebegriffs und geht dabei insbesondere der Frage nach, was es bedeutet, wenn etwas in kritischer Absicht als Bürokratie oder bürokratisch bezeichnet wird. Weshalb wird Bürokratie so oft als etwas Unerwünschtes wahrgenommen? Und was streben diejenigen an, die das Bürokratische durch das Unbürokratische überwinden wollen? Um Antworten auf diese Fragen zu finden, blickt Cancik auch in die jüngere Geschichte des Entbürokratisierungsdiskurses innerhalb der Europäischen Union.

Eine radikal wirtschaftsliberale Spielart der Bürokratiekritik findet sich unter der Regierung Bolsonaro gegenwärtig in Brasilien, der seine Präsidentschaft 2019

4 Max Weber 1972 [1921/22]. Wirtschaft und Gesellschaft. 5., revidierte Auflage. Tübingen: Mohr Siebeck, S. 563. 
mit dem Versprechen angetreten ist, das Land durch Marktöffnung, Privatisierung und Entbürokratisierung nach neoliberalem Bild zu formen. Auf der Agenda weit oben steht dabei die Öffnung der Amazonas-Region für die Agrar- und BergbauIndustrie, die der Umweltminister Ricardo Salles in der Corona-Pandemie noch weiter vorantreiben möchte. Er sieht in der Pandemie die ideale Gelegenheit, Gesetzesänderungen durchzuführen, ohne einen gesellschaftlichen Aufschrei zu verursachen: "Wir haben jetzt die Möglichkeit, da die Presse sich ausschließlich mit Covid-19 beschäftigt, uns das Amazonas-Thema vorzunehmen. Wir haben in diesem Moment die Chance, alle Regelungen zu ändern und die Vorschriften zu vereinfachen. $\aleph^{5}$ Die Corona-Krise und ihre weitreichenden Folgen, das zeigt dieses Beispiel eindrücklich, ist gegenwärtig nicht die einzige Krise, mit der die brasilianische Bevölkerung zu kämpfen hat. Sie macht sich in einem Land breit, in dem ohnehin bereits vieles im Argen liegt. Frédéric Vandenberghe, der seit 2007 als Professor am Institut für Sozial- und Politikwissenschaften der staatlichen Universität Rio de Janeiro arbeitet, diagnostiziert in seinem Essay eine Akkumulation von Krisen, die das Land an den Rand des Zusammenbruchs geführt haben. Neben einer ökonomischen, politischen, institutionellen, Sicherheits-, ökologischen und Gesundheitskrise befinde sich Brasilien nun auch noch in einer militärischen Krise, die den Autor zu einer düsteren Prognose veranlasst: »Mit dem Zulauf zu den Extremen scheint mir Brasilien nunmehr nicht mehr als drei Optionen zu haben: Absetzung des Präsidenten, Staatsstreich oder Bürgerkrieg."

Wie konnte es so weit kommen? Um eine Antwort darauf zu finden, ist es unerlässlich, nach Erklärungen für den Rechtsruck in Brasilien zu suchen, der mit der Absetzung der ehemaligen Präsidentin Dilma Roussef 2016 seine verhängnisvolle Fahrt aufnahm. Dies ist das Anliegen unseres Autors Sérgio Costa, der die politische Transformation Brasiliens in seinem Aufsatz als Ausdruck eines intersektionalen Verteilungskonflikts deutet. Er sieht im gegenwärtigen Rechtsruck den Versuch, die sozialstrukturellen und machtpolitischen Verschiebungen während der Regierungen der Arbeiterpartei zugunsten der Armen und einer neuen Mittelschicht sowie der Schwarzen, Frauen und LGBT-Gruppen rückgängig zu machen und bestehende soziale Hierarchien aufrechtzuerhalten beziehungsweise wiederherzustellen.

Wie steht es aktuell um das krisengebeutelte Land und seinen Präsidenten? Während im Amazonasurwald, im Pantanal und vielen anderen Landesteilen die durch Brandrodungen verursachten Rauchschwaden den Himmel verdunkeln, sterben immer mehr Menschen an Covid-19. Das katastrophale Krisenmanagement der Regierung hat dazu geführt, dass sich das Virus in Brasilien besonders stark und ungehemmt ausbreiten konnte. Anstatt der Pandemie den Kampf anzusagen, behinderte Bolsonaro ihn. Er kritisierte die Abstandsregeln, die viele Städte und Bundesstaaten eingeführt hatten, empfahl die Einnahme von Medikamenten ohne nachgewiesene Wirksamkeit, hielt Massenkundgebungen ab und verhinderte die Maskenpflicht im Einzelhandel, in Schulen und religiösen Einrichtungen.

5 Siehe www.fr.de/wirtschaft/brasilien-corona-krise-regenwald-abholzung-13776889.html (Zugriff vom 22.10.2020). 
Umso erstaunlicher ist es, dass Bolsonaro sich derzeit in einem Umfragehoch befindet. Die Zufriedenheit mit dem Präsidenten ist höher denn je. Der Grund dafür ist mindestens ebenso erstaunlich: Kurz nach dem Ausbruch der Pandemie beschloss die Regierung auf Druck von Opposition und Zivilgesellschaft eine Nothilfe für Bedürftige, von der seit April dieses Jahres 40 Prozent der Erwachsenen profitieren. Sie ist nicht hoch, aber ausreichend, um zu überleben und den Konsum anzukurbeln. Angesichts des steigenden Haushaltsdefizits wurde die Hilfe jedoch im September halbiert, am Ende des Jahres soll sie auslaufen. Bolsonaro hat zwar angekündigt, die Nothilfe im nächsten Jahr in ein neues Sozialprogramm zu überführen, welches das Armutsbekämpfungsprogramm des Ex-Präsidenten Luíz Inácio Lula da Silva ersetzen soll. ${ }^{6}$ Ob der mit neoliberaler Programmatik angetretene Präsident jedoch tatsächlich eine sozialpolitische Wende vollziehen wird oder es sich dabei lediglich um ein (popu)leeres Versprechen handelt, ist alles andere als gewiss. So oder so, der Regenwald wird dadurch nicht gerettet.

Claudia Czingon

6 Siehe www.dw.com/de/corona-macht-bolsonaro-beliebt/a-54795057 (Zugriff vom 22.10.2020). 